\title{
Photonic Crystal Nanocavities and Waveguides
}

\section{A. Scherer, J. Vuckovic, M. Loncar, T. Yoshie, O. Painter Caltech, Pasadena, CA 91125}

Fabrication of optical structures has evolved to a precision which allows us to control light within etched nanostructures. Nano-optic cavities can be used for efficient and flexible concentration of light in small volumes, and control over both emission wavelength and frequency. Conversely, if a periodic pattern is defined in the top semitransparent metal layer by lithography, it is possible to efficiently couple out the light out of a semiconductor and to simultaneously enhance the spontaneous emission rate. Here we demonstrate the use of photonic crystals for efficient light localization and light extraction. 


\title{
Photonic Crystal Nanocavities and Wavegudies
}

\author{
A. Scherer, J. Vuckovic, M. Loncar, T. Yoshie, O. Painter \\ Caltech, Pasadena, CA 91125
}

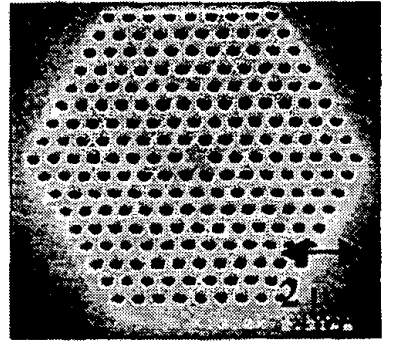

Figure 1. Photonic crystal nanocavity laser structure fabricated in InGaAsP
When combined with high index contrast slabs in which light can be efficiently guided, microfabricated two-dimensional photonic bandgap mirrors provide us with the geometries needed to confine and concentrate light into extremely small volumes and to obtain very high field intensities. Fabrication of optical structures has now evolved to a precision which allows us to control light within such etched nanostructures. Sub-wavelength nano-optic cavities can be used for efficient and flexible control over both emission wavelength and frequency, and nanofabricated optical waveguides can be used for efficient coupling of light between devices. The reduction of the size of optical components leads to their integration in large numbers and the possibility to combine different functionalities on a single chip, much in the same way as electronic components have been integrated for improved Vertical Cavity Surface Emitting Lasers (VCSELs) can be largely attributed to the high precision over the layer thickness control available during semiconductor crystal growth. High reflectivity mirrors can thus be grown with sub-nanometer accuracy to define high-Q cavities in the vertical dimension. Recently, it has also become possible to microfabricate high reflectivity mirrors by creating two- and three-dimensional periodic structures. These periodic "photonic crystals" can be designed to open up frequency bands within which the propagation of electromagnetic waves is forbidden irrespective of the propagation direction in space and define photonic bandgaps. When combined with high index contrast slabs in which light can be efficiently guided, microfabricated two-dimensional photonic bandgap mirrors provide us with the geometries needed to confine and concentrate light into extremely small volumes and to obtain very high field intensities. Here. we propose to use these "artificial" crystals in functional nonlinear optical devices, such as lasers, modulators, add/drop filters, polarizers and detectors.

Fabrication of optical structures has evolved to a precision which allows us to control light within

Figure 2. Modeling results showing a dipole mode in aphotonic crystal cavity. 3-D finite difference time domain model

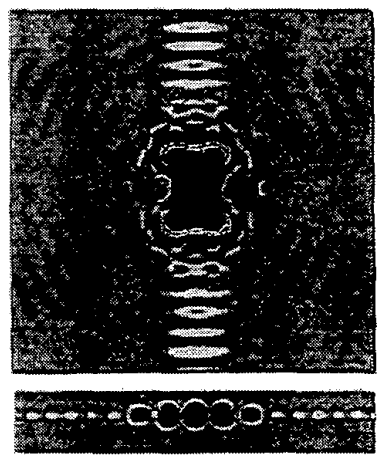
etched nanostructures. For example, sub-wavelength nano-optic cavities can be used for efficient and flexible control over both emission wavelength and frequency. Similarly, nanofabricated optical waveguides can be used for efficient coupling of light between devices. This new capability allows the reduction of the size of optical components and leads to their integration in large numbers, much in the same way as electronic components have been integrated for improved functionality to form microchips. As high-Q optical and electronic cavity sizes approach a cubic half-wavelength the spatial and spectral densities (both electronic and 
optical) increase to a point where the light-matter coupling becomes so strong that spontaneous emission is replaced by the coherent exchange of energy between the two systems.

\section{Surface Plasmon Enhanced Light Emitting Diodes}

We have recently demonstrated a method for enhancing the emission efficiency of light-emitting diodes, by using coupled surface plasmons. The analyzed structure consists of a $90 \mathrm{~nm}$ thick semiconductor layer sandwiched between two silver films. A single $8 \mathrm{~nm}$ thick InGaAs quantum well is positioned in the middle of the semiconductor membrane. The main emission peak is at a wavelength of $990 \mathrm{~nm}$, corresponding to conduction-to-heavy hole $(\mathrm{C}-\mathrm{HH})$ band transitions. Another peak can be



Figure 3. Scanning Electron Micrograph of a surface plasmon enhanced LED showing the metal grating pattern which is used to couple out the radiation. observed at $930 \mathrm{~nm}$, corresponding to conduction-tolight hole $(\mathrm{C}-\mathrm{LH})$ band transitions. If a periodic pattern is defined in the top semitransparent metal layer by lithography (Figure 3), it is possible to efficiently couple out the light emitted from the semiconductor and to simultaneously enhance the spontaneous emission rate. For the analyzed designs, we theoretically estimate extraction efficiencies as high as $37 \%$ and Purcell factors (Fp) of up to 4.5. We have experimentally measured photoluminescence intensities of up to 46 times higher in fabricated structures compared to unprocessed wafers. The increased light emission is due to an increase in the efficiency of the diode and an increase in the pumping intensity resulting from

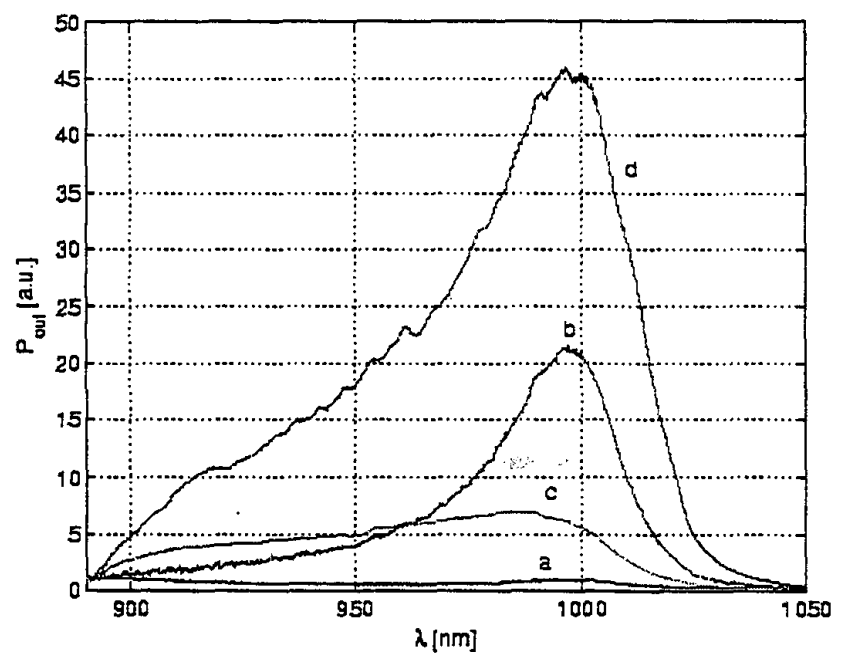

Figure 4. Photoluminescence measurements taken from surface plasmon light emitting diode at various points of the fabrication process trapping of pump photons within the microcavity. The measured photoluminescence spectra are shown in Figure 4. The spectra correspond to: (a) the unprocessed wafer; (b) the half-processed wafer (i.e. $90 \mathrm{~nm}$ thick semiconductor membrane on top of a thick, nontransparent silver layer); (c) the unpatterned metal-clad microcavity (i.e. a semiconductor membrane sandwiched between two metal films, without patterning of the top silver layer) and (d) the fully processed structure where silver stripes are defined in the top silver layer, with the grating periodicity of $250 \mathrm{~nm}$ and the $160 \mathrm{~nm}$ gap between silver stripes. 